\title{
Perceptual bias and response bias in temporal bisection
}

\author{
THOMAS G. RASLEAR \\ Walter Reed Army Institute of Research, Washington, DC
}

\begin{abstract}
In a temporal bisection task with rats, perceptual bias and response bias were simultaneously varied through manipulations of stimulus spacing and the relative probability of reinforcement for correct responses. Both manipulations produced systematic changes in the bisection point. However, only manipulations of relative reinforcement probability produced significant variations in $\mathrm{B}^{\prime \prime}$, a nonparametric index of response bias. This finding shows that the bisection point may be shifted by either a perceptual bias or a response bias. However, in the absence of an index of response bias, such as B", shifts caused by perceptual effects are indistinguishable from those caused by response preferences.
\end{abstract}

Temporal bisection is employed as a behavioral tool to detect the effects of drugs on time perception (Maricq \& Church, 1983; Maricq, Roberts, \& Church, 1981; Meck, 1983). Changes in the bisection point indicate whether drugs and other treatments affect the perception of time directly, by changing the speed of a hypothesized internal clock, or indirectly, by modifying memory processes. However, an important aspect of drug effects on behavior has not yet been evaluated for the bisection task: it is not known whether response bias may influence the bisection point, and thus cause response preferences to be mistaken for true perceptual effects. This is particularly important with respect to some of the drugs and treatments that have been used in the bisection task to date. For instance, amphetamine can produce response stereotypies (Iverson \& Iverson, 1981), inescapable footshock often produces a depression of movement (Anisman, de Catanzaro, \& Remington, 1978), and response perseveration may follow septal or hippocampal lesions (Braggio \& Ellen, 1976; Ellen, Makohon, \& Richardson, 1978). If any of these effects were present in the bisection task, a response preference or bias could change the bisection point and be mistaken for a true perceptual effect.

The bisection task as implemented for animal subjects is different in several respects from the procedure used with human subjects. Human subjects require little, if any, formal training in the task, whereas extensive training is required to ensure that animal subjects are attending to the stimulus dimension of interest and will perform the response(s) from which a bisection point is derived. An

This material has been reviewed by the Walter Reed Army Institute of Research, and there is no objection to its presentation and or publication. The opinions or assertions contained herein are the private views of the author and are not to be construed as official or as reflecting the view of the Department of the Army or the Department of Defense. Requests for reprints should be sent to Thomas G. Raslear, Department of Medical Neurosciences, Division of Neuropsychiatry, Walter Reed Army Institute of Research, Washington, DC 20307-5100. animal analogue of the human bisection task was first described by Boakes (1969), and subsequent research has generally followed his procedures with some minor variations. $^{1}$

An animal bisection experiment has two distinct phases: discrimination training and generalization testing. In the training phase, subjects learn a simple two-choice discrimination between the two stimuli (A and $B$ ) that define the sensory interval to be bisected. Responses on manipulandum $A$ are reinforced on trials in which stimulus $A$ is presented, and responses on manipulandum $B$ are reinforced on trials in which stimulus $B$ is presented. Inappropriate responses are never reinforced. Once an accurate discriminative performance is achieved, the proportion of correct responses that are reinforced is gradually reduced to the level that will be present during generalization testing.

During generalization testing, several "test" stimuli, intermediate in value to the training stimuli, are introduced. Responses in the presence of the "test" stimuli are never reinforced, whereas correct responses in the presence of the training stimuli continue to be reinforced at a level that maintains the same overall probability of reinforcement as existed during the final stage of the training phase. Responses made in the presence of each of the stimuli (test and training) are recorded and used to construct a generalization gradient. The generalization gradient is usually the proportion of response A (or B) that occurred as a function of stimulus magnitude. The proportion of $A$ responses is near 1.0 in the presence of stimulus A and near 0.0 in the presence of stimulus B. The intermediate-value "test" stimuli produce proportions that lie between the two extremes. The bisection point (BP) is commonly taken as the stimulus magnitude that produces a 0.5 proportion of $A$ responses.

As noted above, the potential role of response bias in the animal bisection task has not yet been evaluated. However, it is known that the bisection task, as implemented for humans and animals, is subject to context 
effects or perceptual bias. For example, changes in stimulus context, such as stimulus spacing, stimulus frequency, or stimulus order in time and space, have been shown to affect psychophysical tasks in humans (Helson, 1964). Fagot and Stewart (1970) demonstrated that the BP shifts with changes in stimulus spatial order in a human brightness bisection task, and Raslear has demonstrated that changes in stimulus spacing produce shifts in the BP for rats in auditory bisection (Raslear, 1975) and in temporal bisection (Raslear, 1983) tasks.

The demonstration that manipulations of stimulus context affect the outcome of a psychophysical task can be interpreted in at least two ways, as Helson $(1971$, p. 6) has noted: "Adaptation-level theorists maintain that with changes in stimulation accompanying focal stimuli the quality, magnitude, and other dimensions of the stimuli also change more or less. Critics of AL theory assert that the changes under such conditions reported by the subjects do not reflect changes in perceived attributes but are merely verbal, semantic, or judgmental artifacts." Response preferences are the "verbal, semantic, and judgmental artifacts" of concern in an animal bisection task, so the issue is whether changes in stimulus context produce changes in the BP because the perceived magnitudes of the stimuli have been affected (i.e., perceptual bias) or because of an induced response preference (i.e., response bias).

Most human studies of perceptual bias have not addressed the issue of whether such biases may be explained in terms of response bias as defined by signal detection theory (however, see Hellstrom, 1985). It is possible that manipulations of stimulus frequency merely act to move the criterion in animal as well as in human studies. Changes in stimulus spacing may merely produce changes in the relative reinforcement rates for the two responses in the bisection task. Since responses to the test stimuli are not reinforced in the typical animal bisection procedure, and some of the test stimuli are likely to be poorly discriminated from one of the training stimuli, a smaller proportion of choices on one response lever would be reinforced. This would result in a decrease in responses on that lever and a shift in the BP. The shift would not be a true perceptual effect, but an instance of response bias.

Figure 1 provides a schematic illustration of how perceptual bias, response bias, and a change in sensitivity would influence the psychometric function in a bisection task. The normal psychometric functions are represented as straight lines for ease of presentation. As shown in panel A of Figure 1, a pure perceptual effect (i.e., response bias and sensitivity are unchanged, but the BP has shifted) would cause a bowing of the normal psychometric function without any simultaneous changes in the two anchor or training stimuli. A pure response bias effect (only sensitivity is unchanged) would cause an upward (or downward) shift in all stimuli, as shown in panel B of Figure 1. Finally, a pure change in sensitivity (BP and
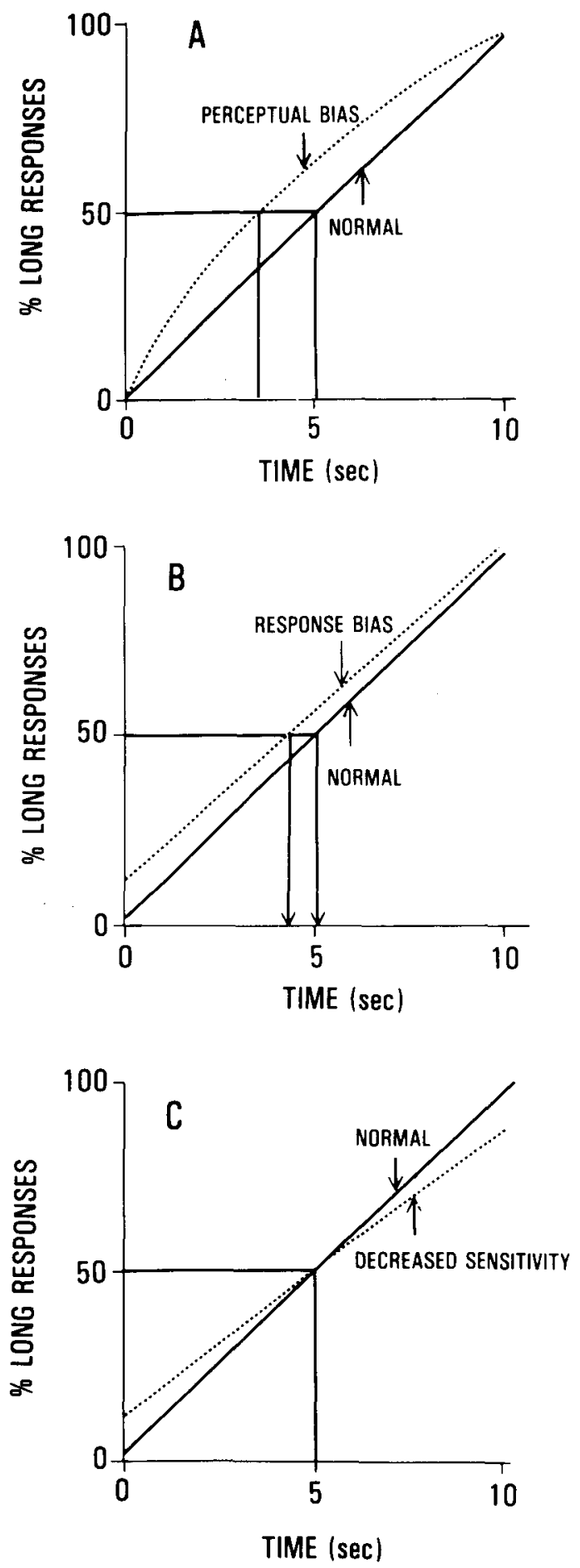

Figure 1. Illustrations of the effects of perceptual bias, response bias, and sensitivity on the psychometric function for bisection. In each case, the normal psychometric function is shown as a straight line for ease of presentation. Percent "long" responses are plotted as a function of linear time, and changes in the bisection point $\mathbf{5 0 \%}$ "long" responses) are shown. 
response bias unchanged) would be seen as a change in slope of the function, as seen in panel $\mathrm{C}$.

Although several studies have demonstrated shifts in the BP with changes in stimulus context (Raslear, 1975, 1983), no study has shown that manipulations of response bias can also affect the BP. The experiment described here addressed this question by simultaneously varying stimulus spacing and the relative probability of reinforcement on the choice levers in a $4 \times 5$ factorial design during generalization testing. Stimulus spacing was varied over a 3.3-to-1 range to manipulate the BP through perceptual bias. Similarly, the relative probability of reinforcement was varied over a 4-to-1 range to manipulate the BP through response bias. Response bias was indexed (independently of the BP) by the nonparametric bias parameter, B" (Grier, 1971). In this way, it was hoped that questions concerning the independence of perceptual and response bias and the measurement of response bias in bisection tasks could be resolved.

\section{METHOD}

\section{Subjects}

Eight male albino specific pathogen-free rats were purchased from Charles River Breeding Labs. The rats were 90-110 days old at the beginning of the experiment. Following a short period of adaptation to the laboratory and individual housing, the rats were reduced to $80 \%$ of their free-feeding weights by restricted daily feeding. The rats were maintained at this weight by suppiementing the food obtained in experimental sessions with Purina Rat Chow. Water was available at all times in the home cages. The animals were housed in individual cages inside a temperature- and humiditycontrolled chamber at an ambient temperature of $23^{\circ} \mathrm{C}$ and $50 \%$ relative humidity. A 12-h: 12-h light-dark cycle (lights on at $0600 \mathrm{~h}$, off at $1800 \mathrm{~h}$ ) was maintained throughout the experiment.

\section{Apparatus}

There were two operant conditioning chambers, previously described by Raslear (1983). Each chamber consisted of a soundand light-attenuating enclosure that contained a Plexiglas and metal cage with a grid floor, two response levers, house and cue lights, a food cup, and a pellet dispenser. Each cage measured $30.5 \mathrm{~cm}$ long $\times 24 \mathrm{~cm}$ high $\times 24 \mathrm{~cm}$ deep. The response levers were mounted $8.25 \mathrm{~cm}$ above the cage floor and $9.5 \mathrm{~cm}$ apart on the same wall as the food cup, which was centered between the levers $3.8 \mathrm{~cm}$ above the cage floor. Two $4.75-\mathrm{W}$ incandescent lamps, mounted at the top of the wall opposite the levers, served as the houselight. The enclosures were ventilated by fans and were located in the same temperature- and humidity-controlled chamber that housed the home cages. A PDP-8/e computer controlled the experiment and recorded data.

\section{Procedure}

General. The experimental session for each subject occurred at approximately the same time each day, 5 days per week excluding holidays. No water was available in the experimental chambers. The subjects were weighed after each session to determine the amount of supplemental food they would receive following the session.

Discrimination training. A two-choice, discrete-trials paradigm was used in which the animals were trained to discriminate between the two durations that defined the temporal interval to be bisected $(0.1$ and $10.0 \mathrm{sec})$. The duration of the houselight served as the discriminative stimulus. Responses were effective only in the $10 \mathrm{sec}$ following the discriminative stimulus. A single correct response terminated the trial and, with a predetermined probability, produced a 45-mg Bio-Serv food pellet, whereas a single incorrect response merely terminated the trial. If no response was made within $10 \mathrm{sec}$, the trial terminated and a null response to that stimulus was recorded. The intertrial interval was $10 \mathrm{sec}$, during which responses had no effect. Each stimulus occurred with a probability of .5 on each trial.

A session consisted of 300 trials, of which the first 20 were used as a warm-up for the animals, with a probability 1.0 of reinforcement for correct responses. Performance on these trials was used by the computer to determine the conditions to which the animals would be exposed in the remaining 280 trials. During discrimination training, if the animals produced a minimum of $90 \%$ correct responses during the warm-up, the remainder of the session consisted of noncorrection-procedure discrimination training with the probability of reinforcement set at the predetermined level. Otherwise, a correction procedure was in effect, in which the discriminative stimulus in whose presence the incorrect response was made was repeated $.02 \mathrm{sec}$ following that response. The probability of reinforcement for correct responses was the same as in the noncorrection procedure.

The probability of reinforcement for correct responses was initially 1.0 and was gradually reduced to .25 for individual animals over the course of $\mathbf{4 0}$ sessions. The criterion for changing reinforcement probability and initiating generalization testing was a minimum of 3 to 5 days under the noncorrection procedure.

Generalization testing. A maintained generalization procedure was used during the test phase. Five new durations, intermediate to the training stimuli, were presented in addition to the two training stimuli. All seven stimuli were presented 40 times per session, once per block of seven trials in a quasi-random order. Responses to the test stimuli were never reinforced, but $90 \%$ of the correct responses to the training stimuli were reinforced to maintain approximately the same probability of reinforcement as in discrimination training.

Two biasing operations-one manipulating stimulus spacing, the other manipulating probabilities of reinforcement during the two training stimuli-were simultaneously manipulated across generalization sessions. Four different stimulus spacings were tested within five different relative reinforcement conditions in a quasirandom order. In addition, one of the $\mathbf{2 0}$ combinations was retested to assess the influence of order effects. Table 1 presents the four stimulus sets that were used and the geometric mean (GM) of the duration of the stimuli in each set. Table 2 presents the five reinforcement probability ratios (RP) and their definitions in terms of

Table 1

Duration of Test Stimuli (in seconds) for Four Stimulus Sets Used in Generalization Tests

\begin{tabular}{|c|c|c|c|c|c|c|c|}
\hline \multirow[b]{2}{*}{ GM } & \multicolumn{7}{|c|}{ Test Stimulus } \\
\hline & "Short" & 1 & 2 & 3 & 4 & 5 & "Long" \\
\hline 0.76 & 0.1 & 0.15 & 0.21 & $\overline{0.46}$ & $\overline{2.19}$ & $\overline{4.68}$ & 10.0 \\
\hline 1.00 & 0.1 & 0.21 & 0.46 & 1.00 & 2.19 & 4.68 & 10.0 \\
\hline 1.77 & 0.1 & 0.46 & 1.00 & 3.00 & 5.05 & 7.75 & 10.0 \\
\hline 2.52 & 0.1 & 1.34 & 3.81 & 5.05 & 6.29 & 8.76 & 10.0 \\
\hline
\end{tabular}

Table 2

Absolute and Relative Reinforcement Probabilities Used in Generalization Tests

\begin{tabular}{|c|c|c|c|c|}
\hline \multicolumn{4}{|c|}{ Absolute } & \multirow{2}{*}{$\begin{array}{l}\text { Relative } \\
\text { (Long/Short) }\end{array}$} \\
\hline $\begin{array}{l}\text { "Short" } \\
\text { Stimulus }\end{array}$ & $\begin{array}{l}\text { "Long" } \\
\text { Stimulus }\end{array}$ & $\begin{array}{c}\text { Training } \\
\text { Stimuli }\end{array}$ & $\begin{array}{c}\text { All } \\
\text { Stimuli }\end{array}$ & \\
\hline 1.0 & 0.5 & 0.75 & 0.21 & 0.50 \\
\hline 1.0 & 0.8 & 0.90 & 0.26 & 0.80 \\
\hline 0.9 & 0.9 & 0.90 & 0.26 & 1.00 \\
\hline 0.8 & 1.0 & 0.90 & 0.26 & 1.25 \\
\hline 0.5 & 1.0 & 0.75 & 0.21 & 2.00 \\
\hline
\end{tabular}


the probabilities of reinforcement for correct responses to each of the training stimuli. Table 2 also shows the average probability of reinforcement for correct responses to training stimuli and to all stimuli. Although the probability of reinforcement in the two most extreme conditions ( $R P=0.5$ and $R P=2.0$ ) for correct responses to training stimuli decreased from 0.9 to 0.75 , the effective probability of reinforcement over all stimuli remained approximately the same.

Table 3 presents the order in which the 21 generalization tests were presented. One of the tests was conducted each day for 5 consecutive days to provide five estimates of each of the dependent variables.

\section{RESULTS}

\section{Data Analysis}

The proportion of "long" responses (responses to the lever that was reinforced for responses to the longduration training stimulus) occurring to each stimulus was computed to provide daily generalization data for each subject. A mean psychometric function (mean proportion of "long" responses as a function of stimulus duration) was then calculated and used to determine the daily BP. The BP was defined as that stimulus duration that produced a 0.5 proportion of "long" responses. It was decided, prior to data collection, to determine the BP by simple graphic interpolation. This was done to ensure that the same method could be used throughout the experiment in the event that an experimental condition produced irregular psychometric functions that could not be analyzed by other methods without serious objections (see Guilford, 1954, pp. 134-135). Previous research (Raslear, 1975, 1983) on stimulus spacing had indicated that this precaution was advisable. Five BPs were obtained in this fashion for each condition of Table 3 (one per day for 5 consecutive days) and served as the basis for analyses of the effects of bias manipulations on the BP.

Response bias was measured by calculating B" (Grier, 1971) from the daily mean psychometric functions using "long" responses to the long training stimulus $(10 \mathrm{sec})$ to define hits $(\mathrm{H})$ and "long" responses to the short training stimulus $(0.1 \mathrm{sec})$ to define false alarms (FA). In a similar fashion, $A^{\prime}$, a nonparametric signal detection measure of sensitivity (Grier, 1971), was also calculated from daily mean psychometric functions. These measures were computed from the formulas:

$$
\begin{aligned}
& A^{\prime}=\left\{\left[(H-F A)+(H-F A)^{2}\right] /[4 H(1-F A)]\right\}+0.5 \\
& B^{\prime \prime}=\left[\left(H-H^{2}\right)-\left(F A-F A^{2}\right)\right] /\left(H-H^{2}+F A-F A^{2}\right)
\end{aligned}
$$

Table 3

Order of Experimental Conditions

\begin{tabular}{rrrrrr}
\hline & \multicolumn{5}{c}{ Relative Probability of Reinforcement } \\
\cline { 2 - 6 } GM & 0.5 & 0.8 & 1.0 & 1.25 & 2.0 \\
\hline 0.76 & 9 & 18 & 6 & 7 & 8 \\
1.00 & 15 & 2 & 1,5 & 3 & 12 \\
1.77 & 17 & 16 & 21 & 19 & 13 \\
2.82 & 10 & 20 & 4 & 14 & 11 \\
\hline
\end{tabular}

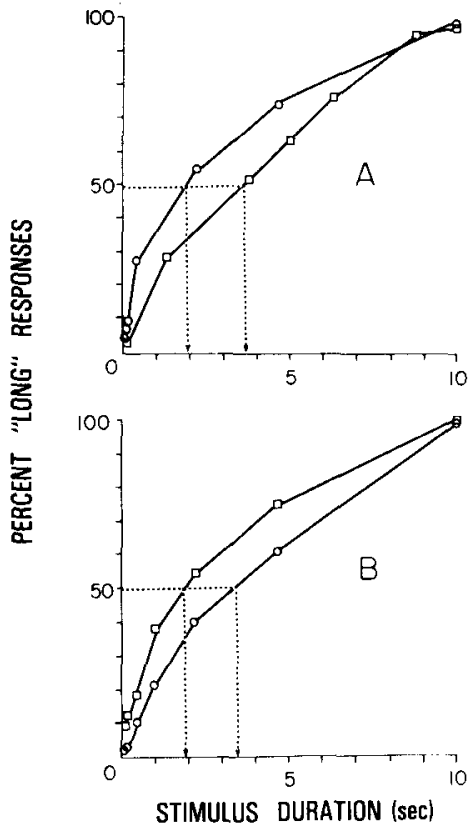

Figure 2. Illustration of the determination of the bisection point, $A^{\prime}$, and $B^{\prime \prime}$ from psychometric functions. Panel A presents psychometric functions for $R P=1.0, G M=0.76$ (circles) and $R P=1.0$, $\mathrm{GM}=2.82$ (squares). Panel $B$ presents psychometric functions for $R P=0.8, G M=1.0$ (circles) and $R P=2.0, G M=1.0$ (squares). In each panel, percent "long" responses are plotted as a function of the stimulus duration.

Five $B^{\prime \prime}$ and $A^{\prime}$ values were thus obtained for each condition of Table 3 .

The method of obtaining the values of BP, B", and A' is illustrated in Figure 2, which presents psychometric functions for four conditions. Panel A shows the percent "long" responses as a function of duration for $R P=1.0$, $\mathrm{GM}=0.76$ (circles) and $\mathrm{RP}=1.0, \mathrm{GM}=2.82$ (squares). The BP is obtained by graphic interpolation, as shown by the dashed lines. $A^{\prime}$ and $B^{\prime \prime}$ are determined by the proportion of "long" responses occurring to the training stimuli. For $\mathrm{GM}=\mathbf{0 . 7 6}$, the hit rate (proportion of "long" responses given the "long" stimulus) is 0.9929 and the false-alarm rate (proportion of "long" responses given the "short" stimulus) is 0.0426 ; for $\mathrm{GM}=2.82$, the hit rate is 0.9809 and the false-alarm rate is 0.0244 . Thus, for the $\mathrm{GM}=0.76$ condition, $\mathrm{BP}=1.9 \mathrm{sec}, \mathrm{B}^{\prime \prime}=$ -.7071 , and $\mathrm{A}^{\prime}=.9874$; for the $\mathrm{GM}=2.82$ condition, $\mathrm{BP}=3.7 \mathrm{sec}, \mathrm{B}^{\prime \prime}=-.12$, and $\mathrm{A}^{\prime}=.9889$.

Similarly, Panel B of Figure 2 shows psychometric functions for $\mathrm{GM}=1.0, \mathrm{RP}=0.8$ (circles), and for $\mathrm{GM}=1.0, \mathrm{RP}=2.0$ (squares). The dashed lines indicate that BP changes from $3.45 \mathrm{sec}$ when $R P=0.8$ to $1.85 \mathrm{sec}$ when $R P=2.0$. Hit and false-alarm rates show whether changes in sensitivity and bias have also taken place (for $\mathrm{RP}=.8, \mathrm{H}=.9879, \mathrm{FA}=.019, \mathrm{~A}^{\prime}=.9921$, $\mathrm{B}^{\prime \prime}=-.2167$; for $\mathrm{RP}=2.0, \mathrm{H}=.9921, \mathrm{FA}=.0926$, $\mathrm{A}^{\prime}=.9745, \mathrm{~B}^{\prime \prime}=-.83$ ).

Previous research on stimulus spacing effects in bisection (Raslear, 1975) has noted that some sets of stimuli 
produce "step-function" generalization gradients. Bisection points obtained from such gradients are artifacts of stimulus spacing because the last stimulus that produced a 0 proportion of "long" responses determines the value of the BP. Step-function gradients were not produced by any of the conditions used in the present study. Manipulations of stimulus spacing and reinforcement probabilities produced orderly changes in the whole generalization gradient similar to that seen in Figure 2.

The effects of stimulus spacing and reinforcement probability ratios on $\mathrm{BP}, \mathrm{A}^{\prime}$, and $\mathrm{B}^{\prime \prime}$ were determined by performing nonparametric two-way analyses of variance, as described by Bradley (1968, pp. 138-141). Except where noted, one-tailed Friedman tests constituted the analyses of variance.

\section{Stability and Sequential Effects}

Over an extended series of tests, time-dependent changes can occur in dependent variables and become confounded with changes produced by experimental manipulations. For instance, gradient steepening, which is common in maintained generalization procedures (see Mackintosh, 1974, pp. 484-542), could gradually change the BP. To assess stability over time, a Daniel's trend test (Bradley, 1968) was performed on BP, B', and A'. The tests showed that there was no reliable trend in any of these measures (for $\mathrm{BP}, \mathrm{B}^{\prime \prime}$, and $\mathrm{A}^{\prime}, \mathrm{r}_{\mathrm{s}}=.09, .17$, and $-.38 ; \mathrm{p}>.05$, two-tailed).

It is also possible that preceding conditions could affect the dependent variables in an experiment that exposes the same subjects to multiple conditions. Since Condition 1 and Condition 5 used the same GM and RP, these two conditions provide information concerning the presence of sequential effects in the experiment. Wilcoxon signed ranks tests performed on the BP, B", and $\mathrm{A}^{\prime}$ measures, however, indicated that there were no reliable differences between the two conditions $(p>.05)$. Therefore, the average of these conditions was used in all further analyses and figures.

\section{The Bisection Point}

Figure 3 presents the mean bisection point as a function of the reinforcement probability ratio (RP) and the stimulus distribution (GM). With the exception of three inversions (at $\mathrm{RP}=.05, \mathrm{GM}=1.0 ; \mathrm{RP}=0.8, \mathrm{GM}=1.77$; $\mathrm{RP}=1.25, \mathrm{GM}=2.82$ ), there are orderly trends in the data with respect to manipulations of the GM and RP, which can be seen more clearly in Tables 4 and 5. Table 4 presents the means of $B P, A^{\prime}$, and $B^{\prime \prime}$ as a function of $\mathrm{GM}$, and Table 5 presents the means of $\mathrm{BP}, \mathrm{A}^{\prime}$, and $\mathrm{B}^{\prime \prime}$ as a function of RP. In general, the BP increases with increases in GM, whereas the BP first increases, then decreases, as RP increases. The nonparametric analysis of variance confirmed these impressions (for GM, $\mathrm{p}<.01$, one-tailed Friedman test; for RP, $p<.01$, onetailed Friedman test) and further indicated that there was a reliable interaction between GM and $\mathrm{RP}(\mathrm{p}<.01$, onetailed Friedman test). The interaction, however, appears

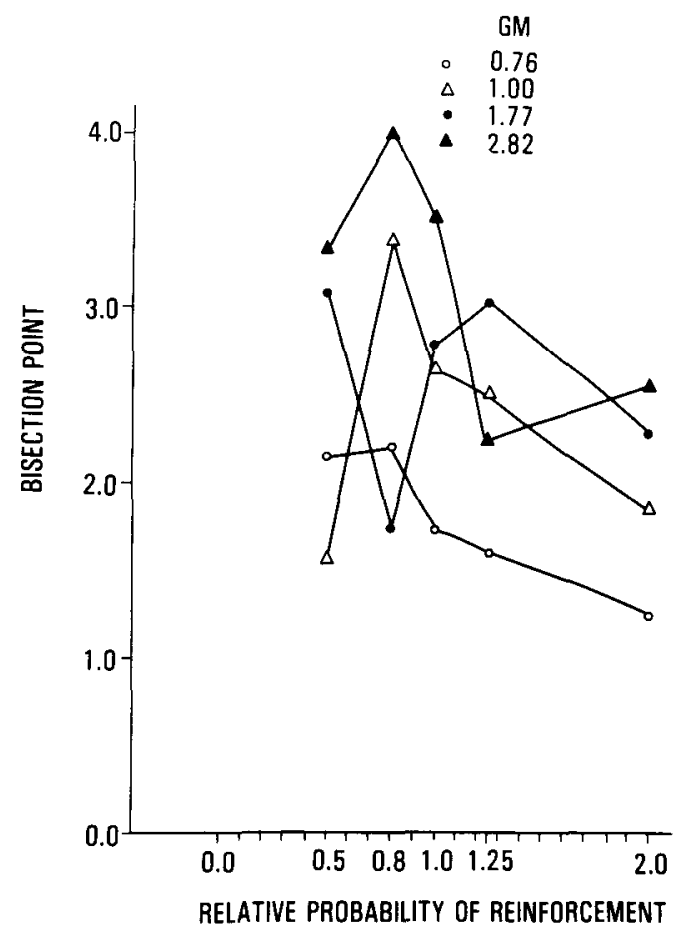

Figure 3. Mean bisection points as a function of the relative probability of reinforcement. The parameter varied within the figure is the geometric mean of the generalization stimulus set.

to be nonsystematic and is probably related to the three data inversions noted above.

\section{Response Bias}

Figure 4 presents the mean $B$ " values as a function of RP and GM. Response bias, as indexed by B", appears to be more clearly affected by variations in RP than by manipulations of GM. Although the range of effect of GM and RP on B" are roughly equivalent (see Tables 4 and

Table 4

Mean Values of Bisection Point (BP), Response Bias (B "), and Sensitivity ( $A^{\prime}$ ) for Four Stimulus Distributions (GM)

\begin{tabular}{lcccc}
\hline & \multicolumn{3}{c}{ GM } \\
\cline { 2 - 5 } & 0.76 & 1.00 & 1.77 & 2.82 \\
\cline { 2 - 5 } & & & & \\
BP & 1.79 & 2.40 & 2.62 & 3.14 \\
B $^{\prime \prime}$ & -.3681 & -.3821 & -.3242 & -.1965 \\
A $^{\prime}$ & .9842 & .9866 & .9809 & .9836 \\
\hline
\end{tabular}

Table 5

Mean Values of Bisection Point (BP), Response Bias (B "), and Sensitivity $\left(A^{\prime}\right)$ for Five Values of Relative Probability of Reinforcement (RP)

\begin{tabular}{lccccc}
\hline & \multicolumn{5}{c}{ Relative Probability of Reinforcement } \\
\cline { 2 - 6 } & 0.5 & 0.8 & 1.0 & 1.25 & 2.0 \\
\hline BP & 2.56 & 2.85 & 2.68 & 2.34 & 2.00 \\
B $^{\prime \prime}$ & -.2762 & -.2998 & -.2446 & -.3535 & -.4145 \\
A $^{\prime}$ & .9831 & .9868 & .9874 & .9833 & .9785 \\
\hline
\end{tabular}




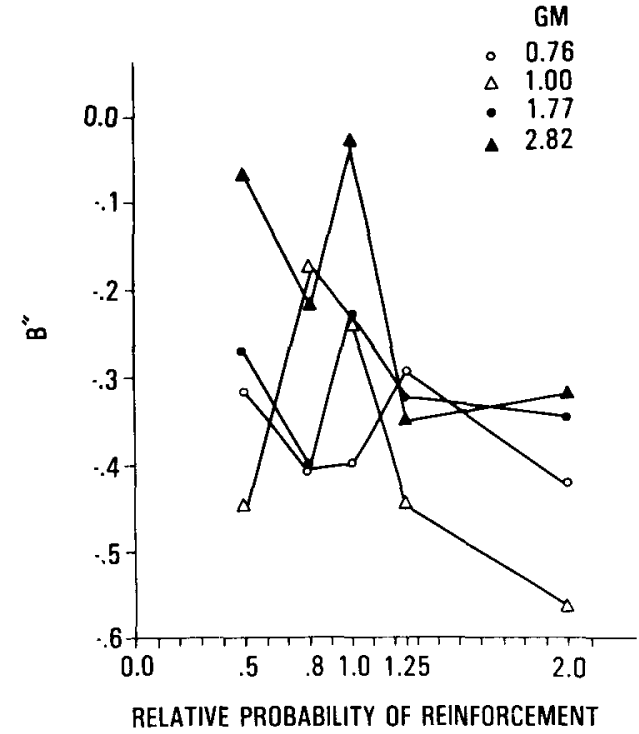

Figure 4. B" as a function of the relative probability of reinforcement. The parameter varied within the figure is the geometric mean of the generalization stimulus set.

5), it can be seen from Figure 4 that there is more variability in B" across manipulations of GM than for RP (for $G M$, the standard error of the mean $B^{\prime \prime}$ is 0.14 ; for $R P$, it is 0.089). Thus, despite several inversions, $B$ " (which takes negative values here) decreases with increases in $R P(p<.05$, one-tailed Friedman test). This is an expected effect of response bias in this experiment. Values of RP $<1.0$ indicate that there is a higher probability of reinforcement for "short" responses, whereas values of RP $>1.0$ indicate that there is a higher probability of reinforcement for "long" responses. Values of $B$ " $<0.0$ indicate a "long" response bias, and if B" $>0.0$, a "short" response bias is present. Thus, as RP increases (payoffs are biased toward "long" responses), B" should decrease (indicating a "long", response bias) and BP should decrease, because the stimulus that received 50\% "long" responses in a zerobias condition would now receive more "long" responses. A shorter duration would receive 50\% "long" responses instead.

Although there was a significant effect on $B$ " of RP, the manipulations of GM $(\mathrm{p}>.05$, one-tailed Friedman test) and the $G M \times R P$ interaction $(p>.05$, one-tailed Friedman test) were both nonsignificant.

\section{Sensitivity}

Figure 5 presents the mean $A^{\prime}$ values as a function of RP and GM. Clearly, variations in the two bias parameters did not have an appreciable effect on sensitivity, as indexed by $A^{\prime}$. The nonparametric analysis of variance revealed no significant effects of experimental manipulations on $\mathrm{A}^{\prime}$ (for $\mathrm{GM}, \mathrm{p}>.05$, one-tailed Friedman test; for RP, $p>.05$, one-tailed Friedman test; for GM $\times R P, p>.05$, one-tailed Friedman test).

\section{DISCUSSION}

\section{Does Response Bias Affect the Bisection Point?}

Although it was recognized that response bias could influence the BP, previous research (Raslear, 1983) did not directly manipulate response bias and failed to detect an effect of response bias during manipulations of stimulus spacing. The present experiment clearly demonstrates that response bias does affect the BP. Manipulations of RP produced changes in BP that are similar to those produced by manipulations of GM in this and other studies (Raslear, 1975, 1983).

\section{Is Perceptual Bias Different From Response Bias?}

Given that both RP and GM influence BP, can it be said that GM manipulations operate by creating a perceptual bias that is different from the response bias created by RP manipulations? Observations of B" indicate that only $\mathrm{RP}$ is effective in causing changes in response bias. There were no reliable effects of GM or of GM $\times$ RP interactions on B". This is consistent with previous work (Raslear, 1983), which found no changes in response bias between stimulus spacing conditions that produced large changes in BP. Since there is no evidence that varying $\mathrm{GM}$ results in reliable changes in response bias $\left(\mathrm{B}^{\prime \prime}\right)$, changing GM is not equivalent to changing RP, which does alter response bias (B"). Thus, the mechanism (perceptual bias) by which GM changes the bisection point must be different from the mechanism operating when RP is varied.

\section{Distinguishing Perceptual from Response \\ Bias Effects on the Bisection Point}

The independence of perceptual and response biases in bisection can be used to distinguish between these effects in experiments, as suggested in Figure 1. For example, Figures 6 and 7 show psychometric functions for several of the conditions in this experiment. In Figure 6, psycho-

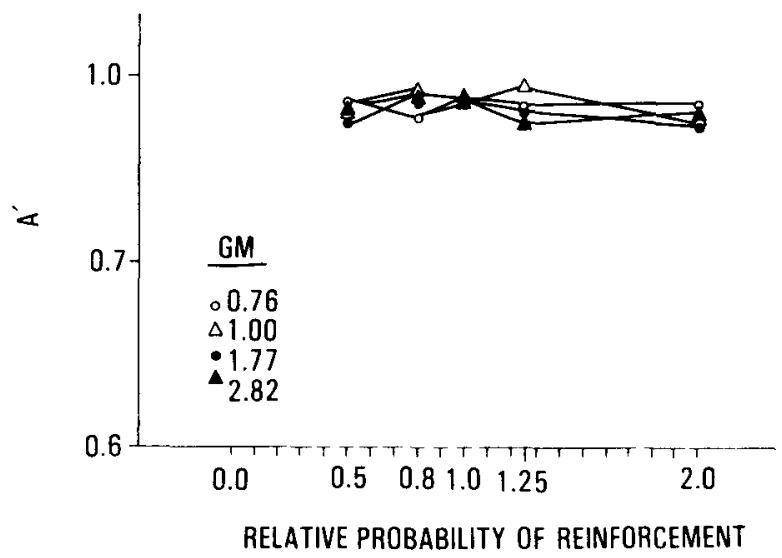

Figure 5. $A^{\prime}$ as a function of the relative probability of reinforcement. The parameter varied within the figure is the geometric mean of the generalization stimulus set. 


\section{$\frac{\mathrm{GM}}{0.76} \quad \frac{\mathrm{BP}(\mathrm{sec})}{1.44} \quad \frac{\mathrm{B}^{\prime}}{.42} \frac{\mathrm{A}^{\prime}}{.98}$ \\ $\triangle 2.82 \quad 2.72 \quad-.32 \quad .98$}

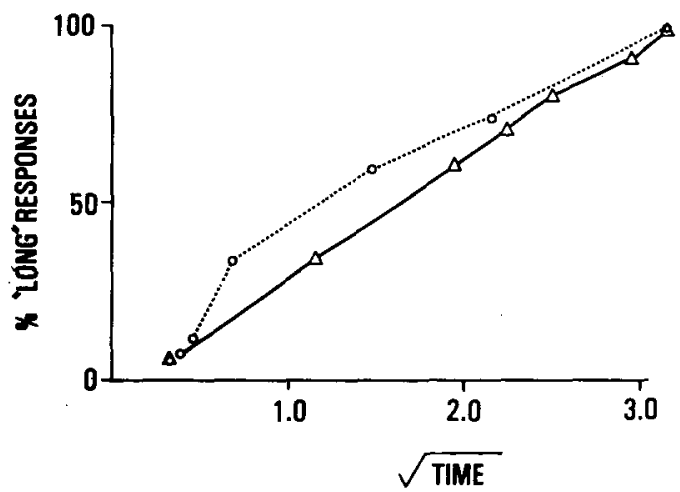

Figure 6. Mean psychometric functions for the $G M=0.76, R P=2.0$ and $G M=2.82, R P=2.0$ conditions. Percent "long" responses are plotted as a function of the square root of time. The functions coincide at the training stimuli, but separate at intermediate stimulus durations. This results in a large change in the bisection point, but relatively small changes in $B^{\prime \prime}$ and $A^{\prime}$. This is an example of perceptual bias in bisection.

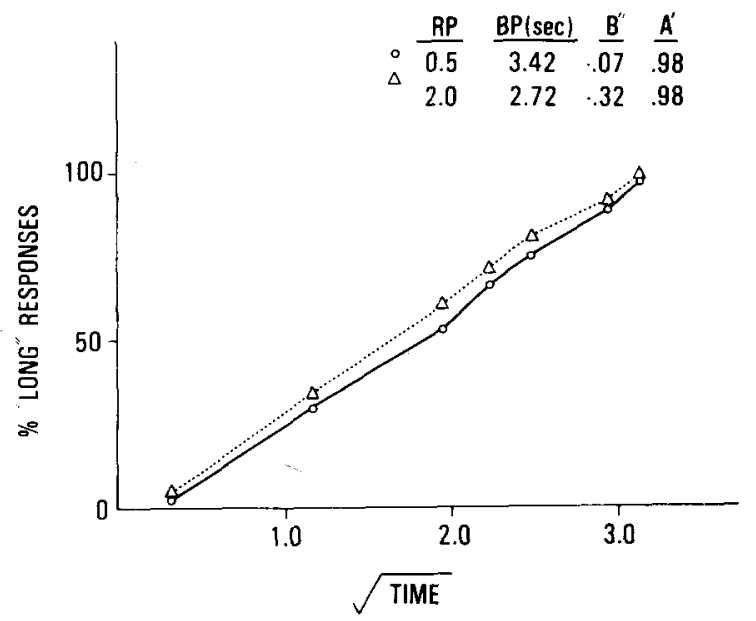

Figure 7. Mean psychometric functions for the $\mathbf{R P}=0.5, \mathbf{G M}=2.82$ and $R P=2.0, G M=2.82$ conditions. Percent "long" responses are plotted as a function of the square root of time. The functions are more parallel to each other than the two functions in the preceding figure. Thus, although the bisection point has still changed, there is a larger change in $B^{\prime \prime}$ and no change in $A^{\prime}$. This is an example of response bias in bisection.

metric functions for the two extreme values of GM used in this experiment are shown. The value of RP is 2.0. The abscissa has been transformed so that the psychometric function for $\mathrm{GM}=2.82$ is a straight line. It is clear that the two end points for $\mathrm{GM}=0.76$ coincide with the two end points for $G M=2.82$. However, the rest of the function for $\mathrm{GM}=0.76$ is bowed. This is a clear example of perceptual bias.

In Figure 7, psychometric functions for the two most extreme values of RP used in this experiment are shown.
The value of GM is 2.82 . The abscissa has been transformed as it was for Figure 6 . Note that the function for $R P=2.0$ lies entirely above the $R P=0.5$ function. The upward shift in the $R P=2.0$ function is expected, since that condition provides a higher reinforcement probability for "long" responses. As indicated above, a "long" bias produces a shift in BP to a smaller value.

It is clear that both perceptual biases and response biases operate in the bisection task. If one examines only the BP values, these two biases can be confounded. Examination of the psychometric functions can reveal the source of the bias influencing the BP, but such visual methods can be extremely misleading when both types of bias are present, or if one of the psychometric functions cannot be transformed to a straight line. Note that in Figures 6 and 7, values for $A^{\prime}$ and $B^{\prime \prime}$ have also been noted. In Figure 6 (where RP is constant), $B^{\prime \prime}$ varies by only 0.1 units, whereas in Figure 7 (where RP has been varied), B" varies by 0.25 units. Thus, B" values, or a similar measure, probably provide the best method to determine if a change in response bias has occurred following an experimental manipulation.

Although manipulations of GM and RP produced significant changes in BP and B", neither variable exerted an influence on $A^{\prime}$. This result indicates that sensitivity, defined in signal detection terms, is independent of both response bias and perceptual bias. This is particularly important, since it is clear that many interesting experimental manipulations could affect sensitivity as well as response and perceptual bias. Other researchers have recognized this possibility and have computed a Weber fraction from the psychometric function to determine if sensitivity has changed between conditions (Meck, 1983). The Weber fraction in such a case is defined as the difference limen divided by the BP. Clearly, such a measure of sensitivity, because it is dependent on BP, is also subject to the influence of perceptual and response biases, and cannot be considered a satisfactory measure of sensitivity. The stability of $A^{\prime}$ in the present experiment strongly suggests that future bisection studies should assess sensitivity by using a signal detection measure rather than one derived from classical psychophysics.

It is clear that the bisection point in animal studies is subject to the influence of bias induced by manipulations of stimulus context (perceptual bias) as well as by manipulations of the payoff matrix (response bias). It is also clear that these variables act in an independent fashion that allow their effects to be distinguished. As was noted earlier, many drugs and other treatments that affect cognitive processes also tend to produce response biases. For this reason, it is important that response bias be measured in bisection experiments to determine the true nature of an observed change in the bisection point.

\section{REFERENCES}

Anisman, H., de Catanzaro, D., \& Remington, G. (1978). Escape performance following exposure to inescapable shock: Deficits in motor response maintenance. Journal of Experimental Psychology: Animal Behavior Processes, 4, 197-218. 
BOAKEs, R. A. (1969). The bisection of a brightness interval by pigeons. Journal of the Experimental Analysis of Behavior, 12, 201-209.

Bradley, J. V. (1968). Distribution-free statistical tests. Englewood Cliffs, NJ: Prentice-Hall.

Braggio, J. T., \& Ellen, P. (1976). Cued DRL training: Effects on the permanence of lesion-induced overresponding. Journal of Comparative \& Physiological Psychology, 90, 694-703.

Ellen, P., Makohon, L., \& Richardson, W. K. (1978). Response suppression on DRL by rats with septal damage. Journal of Comparative \& Physiological Psychology, 92, 511-521.

FAGOT, R. F., \& StewART, M. R. (1970). Test of a response bias model of bisection. Perception \& Psychophysics, 7, 257-262.

GrJeR, J. B. (1971). Nonparametric indexes for sensitivity and bias: Computing formulas. Psychological Bulletin, 75, 424-429.

Guilford, J. P. (1954). Psychometric methods. New York: McGraw-Hill.

Hellstrom, A. (1985). The time-order error and its relations: Mirrors of cognitive processes in comparing. Psychological Bulletin, 97 , 35-61.

HELSON, H. (1964). Adaptation level theory. New York: Harper \& Row.

Helson, H. (1971). Adaptation-level theory: 1970-and after. In M. H. Appley (Ed.), Adaptation-level theory. New York: Academic Press.

IVERSON, S. D., \& IVERSON, L. L. (1981). Behavioral pharmacology. New York: Oxford University Press.

Mackintosh, N. J. (1974). The psychology of animal learning. New York: Academic Press.

MaricQ, A. V., \& ChurCh, R. M. (1983). The differential effects of haloperidol and methamphetamine on time estimation in the rat. Psychopharmacology, 79, 10-15.

MaricQ, A. V., RoberTs, S., \& ChURCH, R. M. (1981). Methamphetamine and time estimation. Journal of Experimental Psychology: Animal Behavior Processes, 7, 18-30.

MECK, W. H. (1983). Selective adjustment of the speed of internal clock and memory processes. Journal of Experimental Psychology: Animal Behavior Processes, 9, 171-201.

Pfanzagl, J. (1968). Theory of measurement. New York: Wiley.

Platt, J. R., \& DAvis, E. R. (1983). Bisection of temporal intervals by pigeons. Journal of Experimental Psychology: Animal Behavior Processes, 9, 160-170.

Raslear, T. G. (1975). The effects of varying the distribution of generalization stimuli within a constant range upon the bisection of a soundintensity interval by rats. Journal of the Experimental Analysis of Behavior, 23, 369-375.

Raslear, T. G. (1983). A test of the Pfanzagl bisection model in rats. Journal of Experimental Psychology: Animal Behavior Processes, 9 , 49-62.

STUB8s, D. A. (1976). Scaling of stimulus duration by pigeons. Journal of the Experimental Analysis of Behavior, 26, 15-25.

\section{NOTE}

1. Because of the dissimilarity in training and testing procedures between the human bisection task and the animal bisection task, it is legitimate to question whether the bisection points obtained in each procedure measure the same thing. Fortunately, this question has been addressed in experiments by Fagot and Stewart (1970) for human bisection and by Raslear (1983) for animal bisection. Both procedures have been found to be consistent with the Pfanzagl (1968) bisection model. In that model, it is assumed that subjects judge distances, rather than ratios, between stimuli. The model allows the construction of a valid interval scale from bisection points if the axioms of the model are satisfied. Thus, bisection points obtained from animal subjects in the fashion described above are functionally equivalent to bisection points obtained in the standard human experiment. Other animal "bisection" procedures (e.g., Stubbs, 1976; Platt \& Davis, 1983), however, have not been similarly tested and may not produce valid bisection points as defined by the Pfanzagl model. Thus, it is not possible to compare the results obtained with those procedures with human bisection data or with data obtained with the procedures used in this and similar experiments.

(Manuscript received March 26, 1984 : revision accepted for publication August 28, 1985.) 